\title{
A pilot program at the worksite to reduce adverse self-medication behaviors
}

\author{
This article was published in the following Dove Press journal: \\ Patient Preference and Adherence \\ 19 August 2009 \\ Number of times this article has been viewed
}

\author{
Patricia J Neafsey ${ }^{1,2}$ \\ Gregory Lutkus ${ }^{2}$ \\ Jessica Newcomb ${ }^{2}$ \\ Elizabeth Anderson ${ }^{1,2}$ \\ 'Center for Health Intervention \\ and Prevention (CHIP); ${ }^{2}$ School \\ of Nursing, University of Connecticut, \\ Storrs, CT, USA
}

Correspondence: Patricia J Neafsey Unit 2026, 23I Glenbrook Rd, School of Nursing and Center for Health Intervention and Prevention (CHIP), University of Connecticut, Storrs, CT 06269, USA

$\mathrm{Tel}+\mathrm{I} 8604860508$

Fax + I 860486000 I

Email patricia.neafsey@uconn.edu

\begin{abstract}
A Next Generation Personal Education Program (PEP-NG) that captures self-reported medication behaviors and delivers a tailored educational intervention on a touchscreen interface was piloted with 11 adults with hypertension, aged 45-60 years, in a worksite setting. A time series design with multiple institution of treatment (four visits over three months) was employed. Blood pressure (BP), self-medication behaviors, self-efficacy, and knowledge for avoiding adverse self-medication behaviors were assessed at each of four visits. Satisfaction was assessed once at visit 4 . Measures pre-PEP (visit 1 ) to visit 4 were compared with paired $t$-tests. The adverse self-medication behavior risk score decreased significantly from visit 1 to visit 4 ( $p<0.05$ ) with a medium effect size. Both knowledge and self-efficacy for avoiding adverse self-medication behaviors increased significantly $(\mathrm{p}<0.05)$ with large effect sizes. All six participants not at BP goal $(<140 / 90 \mathrm{mmHg})$ on visit 1 were at goal by visit 4 . User satisfaction was high as assessed by both quantitative measures and qualitative interviews. These positive results suggest the PEP could play a central role in worksite wellness programs aimed at workers with hypertension.
\end{abstract}

Keywords: hypertension, worksite, information technology, tailored intervention

\section{Introduction}

Over 72 million Americans (33\%) have hypertension and another 31\% of Americans are prehypertensive. ${ }^{1, a}$ Individuals who are normotensive at age 55 are estimated to have a $90 \%$ future lifetime risk for developing hypertension. ${ }^{2}$ Hypertension results in more visits to providers than any other condition and, despite frequent health care visits, patients with hypertension often do not achieve target blood pressure (BP) readings. ${ }^{3-7}$ Nurse-run worksite wellness programs offer an opportunity for BP monitoring and educational interventions targeted to adults with, or at risk for, hypertension. The purpose of this study was to pilot the next-generation of a network-based Next Generation Personal Education Program (PEP-NG) that captures the user's selfreported medication behaviors and delivers a tailored educational intervention aimed at improving medication adherence and reducing adverse self-medication behaviors in workers aged $45-60$ years.

\section{Background}

Poor adherence to antihypertensive regimens is often cited as the principal reason for inadequate BP control and has been associated with preventable adverse drug

${ }^{a}$ Hypertension is defined as a systolic blood pressure (SBP) $>139 \mathrm{~mm} \mathrm{Hg}$ or a diastolic blood pressure (DBP) $>89 \mathrm{~mm} \mathrm{Hg}$. Prehypertension is defined as a SBP of $120-139 \mathrm{~mm} \mathrm{Hg}$ or a DBP of 80-89 mm $\mathrm{Hg}{ }^{1}$ 
events and hospitalization at an estimated annual cost of over US\$100 billion. ${ }^{1}$ Patient adherence is greatest five days pre- and five days post-appointment with the health care provider and usually tapers off significantly within 30 days: the so called "white coat adherence." A Authors of a Cochrane systematic review of recent clinical trials aimed at improving patient adherence to antihypertensive therapy concluded that none of the trials have yet demonstrated large long-term improvements in either adherence, provider visits, or health outcomes. ${ }^{9}$ For example, in two recent studies of US veterans with hypertension, ${ }^{10,11}$ aged 30-87 years, patient education in the form of phone calls and teaching modules did increase patient achievement of BP goals but in a similar study adherence fell to pre-intervention levels when the intervention was discontinued. ${ }^{12}$

In addition to poor adherence to antihypertensive regimens, adverse self-medication behaviors with over-the-counter (OTC) medications, supplements, and alcohol can conflict with antihypertensives and contribute to poor BP control and associated costs. ${ }^{13-17}$ Poly-pharmacy issues arise because often patients with hypertension have comorbid diseases (eg, osteoarthritis) and select an inappropriate OTC analgesic such as nonsteroidal anti-inflammatory drugs (NSAIDs). NSAIDS (eg, ibuprofen) counteract the efficacy of antihypertensive agents and increase BP when taken concurrently. ${ }^{18,19}$ They also antagonize the antiplatelet effects of low-dose aspirin. $^{20}$

Addressing both adherence and adverse self-medication practices is an important step toward reducing the risk of potential adverse drug interactions (PADI). Failure to identify and remediate poor adherence and adverse self-medication behaviors often results in intensified pharmacotherapy with increased doses of antihypertensives and additional antihypertensive agents. This increases the overall cost of treatment as well as escalates the risk of adverse antihypertensive drug side effects. ${ }^{21,22}$

An evidence-based mechanism to maximize access to medication instruction and provider advice is critical to maintaining BP control in patients with hypertension. ${ }^{6,16,23}$ Behavior change stems not only from an increase in knowledge, but an increase in one's perceived ability to apply that knowledge correctly, termed self-efficacy by Bandura. ${ }^{24}$ Our previous educational interventions aimed at increasing older (aged 60 years and over) adults' knowledge and self-efficacy for avoiding adverse self-medication behaviors, demonstrated positive clinical outcomes in terms of improved BP control, and reduced self-report of adverse self-medication behaviors. ${ }^{25-27}$ Review of the literature concerning educational intervention programs for adults with hypertension indicates that there are gaps for persons in the age range of 45-60 years. As this age group has been found to have a lower adherence to antihypertensive regimens than older adults, ${ }^{28}$ interventions aimed at workers with hypertension could help improve clinical outcomes. Results of a pilot test of the PEP-NG with adults with hypertension, aged 45-60, at a university worksite are reported herein.

\section{PEP-NG}

The Personal Education Program (PEP) is an educational intervention previously been shown to be effective in improving knowledge and self-efficacy and reducing adverse self-medication practices in older adults with hypertension. ${ }^{25,26}$ The PEP was enhanced to PEP-NG by incorporating the outcome instruments (previously administered by paper and pencil, eg, demographics, medication use, knowledge, self efficacy, satisfaction) in the interface. The PEP-NG is a risk profiling system that captures and assesses complex self-medication behaviors of patients and assesses their related knowledge and correct medication-taking self-efficacy. Patients access the program via a wireless tablet personal computer (PC) and a stylus interface to answer a set of medication regimen (prescription and OTC) and selfmedication practice questions. A rules engine selects three adverse self-medications behaviors (with the highest risk scores) and delivers tailored interactive educational content including "medicine facts," animations that illustrate the consequences of the adverse behaviors identified, "what you can do" offering corrective strategies, and interactive questions that allow the user to apply information learned. If fewer than three adverse behaviors are identified, the PEP-NG delivers a set of up to three default statements dealing with medication adherence, OTC pain relievers (that can be safely taken with antihypertensives), and dangers of combining different types of pain relievers (prescription or OTC). Summaries of self-reported symptoms, medication use (including frequency/time), adverse self-medication behaviors (along with a thumbnail illustration from the animations), and corrective strategies are printed for the user to take home for self-study. These summaries are also available to the health care practitioner prior to the visit for use in providing corrective or reinforcing guidance, and in oversight of adherence behaviors for improved outcomes.

A detailed description of the PEP and PEP-NG development, results of formative evaluation during development and formal usability testing with older adults and primary care providers (advanced practice nurses) have been published 
elsewhere. ${ }^{29-32}$ Text (written at a grade 6 Flesch-Kincaid reading level) and background colors, contrast, graphics and animation style (including speed of the display, object movements and animation sequences) suit the visual and cognitive characteristics of older adults. ${ }^{29-31,33}$ Extrawide scroll bars and drop-down menus (displayed in blocks of eight lines) ease use for those with stiff joints and/or fine tremor and an animated clock enables the user to easily select the time, frequency, and dosage of medication. ${ }^{30}$

An Access (Microsoft, Redmond, WA, USA) database receives the entered data which includes user, site and provider identity codes, health literacy score, BP (measured and entered by the provider), demographic data, medical conditions, patient reported symptoms, knowledge, selfefficacy, and medication use (including frequency and time of administration). The self-medication behaviors are scored according to a previously validated weighting scheme. ${ }^{26}$ User action is date/time-stamped. Data is transferred to the database via a virtual private network, which meets or exceeds the Health Insurance Portability and Accountability Act (HIPAA) requirements and the European Union Directive 95/46/EC and in accordance with International Organization for Standardization (ISO 9100) international standards. ${ }^{34-37}$

Results of formal usability tests suggest that the final PEP-NG prototype permitted older adult users to navigate the PEP with minimal errors and subject burden with a mean time for interface use $(\mathrm{N}=10)$ of $33.08 \pm 7.65$ minutes. Ratings for system usefulness and satisfaction with the PEP were high for both older adult users and advanced practice nurses. ${ }^{31,32}$

\section{Methods}

The PEP-NG was beta-tested in a worksite setting with 11 individuals with hypertension (aged 45-60 years) over a four visit, three-month period. The specific aims were for participants to: 1) achieve target BP readings; 2) increase knowledge of potential adverse self-medication practices as measured on the knowledge score; 3 ) increase self-efficacy for avoiding adverse self-medication practices as measured on the self-efficacy score; 4) reduce selfreported adverse self-medication behaviors as measured on the adverse self-medication behavior risk score; and 5) demonstrate satisfaction using the PEP.

\section{Participants}

The University Human Subjects Review Board approved the study and all members of the research team completed online research ethics training. All university nonfaculty and nonprofessional staff received recruitment flyers for the study. The study was conducted in a private office in a university research center that houses interdisciplinary health research studies. A student nurse researcher met with each potential participant to describe the study, obtain informed consent, and assess participants for inclusion criteria. Participant inclusion criteria were: 1) not previously involved in a PEP or PEP-NG study, 2) at least age 45 but not more than age 60 (by self-report), 3) a health literacy score of at least 44 (6th grade) as measured by the Rapid Estimate of Adult Literacy in Medicine (REALM) tool, ${ }^{38} 4$ ) taking prescribed antihypertensive medication, and 5) independent physical and cognitive functioning including the ability to: a) perform telephone, shopping, travel arrangements, medication taking, and manage finance activities independently on the Instrumental Activities of Daily Living Scale, ${ }^{39}$ b) answer six of 10 items on the Short Portable Mental Status Questionnaire, ${ }^{40}$ and c) be living independently. Participants also needed to demonstrate a visual acuity of at least 20/100 (with corrective lenses if needed). Participants were requested not to participate in another research study related to their health while enrolled in this one.

Participant information was coded by a random ID number selected by the student nurse researcher from a computer-generated list of random numbers. The faculty researchers analyzing the data could not trace a participant ID to identify a specific patient or link private health information to study participants. Only the student nurse researcher had access to the participant name and ID. The tablet PCs were set up so that the PEP-NG was the only site that could be accessed on the tablet (ie, no other Internet connection was available).

\section{Measures}

The knowledge, self-efficacy, adverse self-medication behavior risk and satisfaction scales were previously validated and their psychometric properties described. ${ }^{14,25,26,30}$ The knowledge scale has 14 multiple-choice items, each with one correct response and three distracters. The Knowledge score is the percent correct. Items test both knowledge and application concerning potential interactions with antihypertensives following self-medication with OTC agents, supplements, or alcohol.

The self-efficacy scale is a 12-item instrument with statements related to confidence in selecting appropriate OTC agents and supplements and avoiding drug interactions arising from self-medication behaviors. It has five-point selfreport response categories that range from 1, "Not sure" to 5, "Totally sure." Responses are summed and divided by the 
number of items answered, so that the overall score is not affected by omitted items and is expressed in the original fiv-point metric.

Adverse behaviors are identified from questions that address use (in the past month) of medications to treat high BP and use of OTC agents, supplements, and alcohol for problems often self-managed with nonprescription agents (eg, pain, fever, colds or sinus, allergies, sleep, indigestion, gas, constipation). Users are also asked if they drank alcoholic beverages, smoked or used nicotine, or took any vitamin or mineral supplements (including what, when and how frequently each was taken). A an expert panel (following a modified Delphi method) rated a list of self-medication behaviors on a five-point scale from 1, "Very unlikely" to 5, "Very likely" to cause an adverse outcome. The mean expert rating was used to determine the importance weight for each adverse behavior in the rules engine. The adverse self-medication behavior risk score is the weighted sum of the scores for the adverse behaviors identified.

The satisfaction scale is a 14-item instrument. Eight items address the ease of program use, program content, and suitability of program content, and six items address the perceived likelihood of making behavior change following program use. The five-point Likert-type scale ranges from 1, "Strongly disagree" to 5, "Strongly agree." Ratings are summed and divided by the number of items answered, so that the overall satisfaction scale is not affected by omitted items and is expressed in the original five-point metric.

\section{Procedure}

The PEP-NG was piloted in a university worksite setting from January until June, 2008, using a time series design with multiple institution of treatment (use of the PEP-NG on four visits over three months). Two student nurse researchers were trained to follow the study protocol and their skill in taking BP measurements (with both large and small cuffs $)^{41}$ was verified by a master's prepared registered nurse (MS, RN) in the graduate advanced practice nursing program. They were supervised by a board-certified APRN and director of the university graduate adult primary care program that prepares students for advance practice as adult nurse practitioners with an APRN license.

Each participant met with one of two student nurse researchers once a month for four months in a private conference room in the university interdisciplinary health research center. Participants brought all of their medications (including supplements) to each visit. BP was recorded at the beginning of each visit. On first visit, participants used the
PEP-NG to complete the demographic questionnaire and all of the remaining scales except the satisfaction instrument. The demographic questionnaire was omitted on subsequent visits. At end of PEP-NG use on the fourth visit, participants completed the patient satisfaction instrument on the PEP-NG in addition to the other scales. After each PEP-NG use, the participant met with the student nurse researcher for approximately 10 minutes to go over the printout that listed symptoms, reported adverse self-medication behaviors, and corrective strategies suggested by the PEP-NG. Each participant was given a US\$10 grocery gift card at the end of each of the first three visits and a US\$25 grocery gift card at the end of the 4th visit to compensate for their time in the study.

Following the four visits, participants were invited to contribute in follow-up interviews. Six responded to the invitation and agreed to be interviewed. Interviewed participants were given an additional US\$10 grocery gift card to compensate for their time in the interview. A student nurse researcher met individually with each participant for a 20 minute interview. With a set of 15 open-ended questions, participants were asked about their prior computer usage, their self-perceived knowledge about their medications, their confidence in taking their medications as prescribed, changes in their medication usage during the study, and ideas on how to improve the PEP-NG program. All interviews were tape recorded and the data were later transcribed verbatim by the student nurse researcher/interviewer.

\section{Data analysis}

SAS software (v. 9.1.3; SAS Institute, Cary, NC, USA) was used for data analysis. Descriptive statistics were tabulated for user age, education, health literacy score, knowledge score, self-efficacy score, adverse self-medication behavior risk score, BP, and satisfaction scores. Correlations were conducted between user age, education, and health literacy score and outcome measures of BP and knowledge, self-efficacy and self-medication risk scores to determine if the PEP-NG outcomes were associated with user demographic variables. Outcome measures were also correlated with each other to determine if knowledge and/or self-efficacy was related to self-medication behaviors and if self-medication behavior was related to $\mathrm{BP}$.

We had missing data for one participant on visit 2 who was unable to make the appointment and missing electronic data on visit 3 (due to electrical connectivity issues) for one participant (we did have all BP data for this participant). Consequently, we employed paired $t$-tests to analyze the data on visits 1 and 4 in this small scale, exploratory pilot. 
An a priori alpha for statistical significance was set at 0.05 . Effect sizes (Cohen's $d$ ) were calculated using the standard deviations comparing visit 1 with visit $4 .{ }^{42}$

Content analysis guided analysis of the interview data. ${ }^{43}$ This qualitative research method involves identifying, categorizing, and labeling the patterns in the data. Transcripts were reviewed in detail. Recurring ideas were analyzed and sorted into categories.

\section{Results}

\section{Participant characteristics}

Fifteen participants (12 women, 3 men) responded to the flyers to participate in the study. Three women dropped out of the study before the consent process; one man dropped out of the study following the consent process, but before visit 1 . Personal time constraints were cited as the reasons for not following through on study participation. The final sample consisted of 11 participants ( 9 women, 2 men), all of whom completed the study. All of the participants reported they were Caucasian; one participant indicated he/she was also Native American. The mean age was $55.5 \pm 2.12$ (range 53-57) years. The mean length of education was $15.5 \pm 0.7$ years. All participants had some post-high school education or training and three were college graduates. All REALM scores were 66 out of a possible score of 66 , thus all were able to read at high school level. Ten participants reported that their primary care provider is a doctor and one reported that a nurse practitioner is the primary care provider. All of the participants reported they were PC and Internet users. Eight participants reported using a PC daily and three reported using a PC five days per week. Reported PC use averaged 5.38 hours per day (range 3-6 hours).

At the beginning of the study, 10 of the 11 participants rated their health over the past year as 4 ("good"), one rated health as a 3 ("average") on a five-point scale. The risk of a PADI is increased in individuals having three or more chronic illnesses, taking five or more medications per day, with more than 12 medication doses per day, a history of nonadherence, or taking a drug requiring therapeutic monitoring. ${ }^{44}$ Ten of the participants (91\%) in this study were at risk for a PADI on visit 1 . Four (36\%) reported having three or more chronic illnesses (range 1-5) and two of these reported taking five or more prescription medications a day. The average number of prescription medications taken daily was $2.55 \pm 1.24$ (range 1-6) with two (18\%) taking five or more prescription medications a day. When OTC medications and vitamin/mineral/herbal supplements were included, the average number of medications taken daily in this study was $9.36 \pm 7.78$ (range 3-31) with nine of the $11(82 \%)$ participants taking five or more agents daily and 10 participants $(91 \%)$ taking 12 or more medication doses per day. Ten (91\%) of the participants reported consuming an alcoholic beverage daily. Three of the four participants who reported three or more chronic illnesses and took five or more prescription medications daily and took more than 12 medication doses per day were not at the Seventh Report of the Joint National Committee on Prevention, Detection, Evaluation, and Treatment of High Blood Pressure (JNC-7) BP target on visit 1. Two of these three participants also reported less than daily adherence with their antihypertensive medications.

Table 1 shows the study outcome results for BP, knowledge, self-efficacy and adverse self-medication behavior risk scores.

\section{Blood pressure}

The JNC-7 goal of $<140 / 90 \mathrm{~mm} \mathrm{Hg}^{1,4,45}$ was met by five participants (45\%) on visit 1 and all 11 participants on visit 4 , thus the aim for all particpants to attain the JNC-7 goal by the end of the study was achieved. The mean decline in systolic BP was $-9.27 \pm 15.85 \mathrm{~mm} \mathrm{Hg}(\mathrm{p}=0.0811)$ and the mean decline in diastolic BP was $-4.0 \pm 8.5 \mathrm{~mm} \mathrm{Hg}(\mathrm{p}=0.151)$ from visit 1 to visit 4 . Neither decline in BP was statistically significant (see Table 1).

BP declined over the four visits for all of the $6(55 \%)$ participants not at the JNC-7 target of $<140 / 90 \mathrm{~mm}$ upon study entry. For these participants, the mean preintervention (visit 1) systolic BP was $149.3 \pm 12.75$ and the mean systolic BP on visit 4 was $130.0 \pm 15.8$, a statistically

Table I Results of PEP-NG beta test in workers with hypertension

\begin{tabular}{|c|c|c|c|c|c|}
\hline Outcome & Pre-visit \# I & Visit \#4 & $\mathbf{t}^{\mathrm{b}}$ & $p$ & Cohen's d \\
\hline Variable $^{a}$ & $\begin{array}{l}\text { Mean } \\
\text { (SD) }\end{array}$ & $\begin{array}{l}\text { Mean } \\
\text { (SD) }\end{array}$ & & & \\
\hline Systolic BP (mm Hg) & $\begin{array}{l}137.1 \\
(17.81)\end{array}$ & $\begin{array}{l}127.8^{c} \\
(13.01)\end{array}$ & 1.94 & 0.0811 & -0.60 \\
\hline Diastolic BP (mm Hg) & $\begin{array}{l}87.45 \\
(10.04)\end{array}$ & $\begin{array}{l}83.45^{\circ} \\
(3.80)\end{array}$ & 1.55 & 0.151 & -0.53 \\
\hline Knowledge score \% & $\begin{array}{l}44.15 \\
(20.16)\end{array}$ & $\begin{array}{l}62.70 \\
(21.36)\end{array}$ & 3.28 & 0.0112 & 0.89 \\
\hline Self-efficacy score & $\begin{array}{l}2.08 \\
(0.6 I)\end{array}$ & $\begin{array}{l}3.28 \\
(0.52)\end{array}$ & 6.55 & 0.0002 & 2.12 \\
\hline $\begin{array}{l}\text { Adverse self- } \\
\text { medication behavior } \\
\text { risk score }\end{array}$ & $\begin{array}{l}17.45 \\
(10.23)\end{array}$ & $\begin{array}{l}12.18 \\
(5.32)\end{array}$ & 1.82 & 0.048 & 0.66 \\
\hline
\end{tabular}

Notes: ${ }^{\mathrm{n}} \mathrm{n}=1 \mathrm{I} ;{ }^{\mathrm{b}} \mathrm{d} f=10$; $\mathrm{c}$ All $\mathrm{I}$ participants met the JNC-7 goal by visit 4 . 
significant decrease $(\mathrm{t}=3.18, d f=5 ; p=0.0246)$. The mean pre-PEP (visit 1) diastolic BP was $94.33 \pm 6.37$ and the mean diastolic BP on visit 4 was $85.66 \pm 3.66$, also a statistically significant decrease $(\mathrm{t}=2.89, d f=5, p=0.0341)$. The mean decline in systolic BP for the six participants not at goal at visit 1 was $-19.33 \pm 14.89 \mathrm{~mm} \mathrm{Hg}$ with a large effect size (Cohen's $d=-1.34, r=-0.56$ ). The mean decline in diastolic BP was $-8.66 \pm 7.33 \mathrm{~mm} \mathrm{Hg}$ from visit 1 to visit 4 and was accompanied a large effect size (Cohen's $d=-1.67$, $r=-0.64)$ (see Table 2).

\section{Knowledge and self-efficacy}

Results of a paired samples $t$-test revealed that the increase in the knowledge score was statistically significant with a medium effect size. The increase in self-efficacy scores was statistically significant with a large effect size. knowledge and self-efficacy scores were not significantly correlated with each other.

\section{Behavior risk score}

The decline in the adverse self-medication behavior risk scores was statistically significant with a medium effect size. When asked how often they took their antihypertensive medication, two participants (18\%) responded either "less than daily" or "when I remembered to take it" on at least one visit; both of these were above the JNC-7 goal pre-visit 1. The most common reported adverse self-medication behavior on visit 1 was taking an NSAID that can elevate BP and counteract the

Table 2 Mean blood pressure $(\mathrm{mm} \mathrm{Hg})$ values

\begin{tabular}{|c|c|c|}
\hline \multicolumn{3}{|c|}{ All participants $(\mathbf{N}=I I)$} \\
\hline \multirow[t]{3}{*}{ Visit } & \multicolumn{2}{|c|}{ Blood pressure } \\
\hline & Systolic & Diastolic \\
\hline & Mean (SD) & Mean (SD) \\
\hline 1 & I37.I (17.80) & $87.5(10.0)$ \\
\hline 2 & $128.5(14.34)$ & $89.7(10.8)$ \\
\hline 3 & I29.1 ( 11.32$)$ & $84.9(10.6)$ \\
\hline 4 & $127.8(13.0)$ & $83.4(3.8)$ \\
\hline \multicolumn{3}{|c|}{ Participants not at goal $(<\mid 40 / 90)$ on Visit I $(N=6)$} \\
\hline \multirow[t]{3}{*}{ Visit } & \multicolumn{2}{|c|}{ Blood pressure } \\
\hline & Systolic & Diastolic \\
\hline & Mean (SD) & Mean (SD) \\
\hline I & $149.3(12.7)$ & $94.3(6.3)$ \\
\hline 2 & I34.7 (I5.5) & $96.16(6.3)$ \\
\hline 3 & $129.0(13.7)$ & $88.3(11.1)$ \\
\hline 4 & $130.0(15.8)^{*}$ & $85.6(3.6)^{*}$ \\
\hline
\end{tabular}

Note: *Significantly different from visit I $(p<0.05)$. efficacy of antihypertensives and low-dose aspirin (64\% of participants, $67 \%$ of those not at JNC-7 goal). The second most common behavior was taking a decongestant that can elevate $\mathrm{BP}^{46}$ ( $54 \%$ of participants, $50 \%$ of those not at JNC-7 goal). One of the participants not at the JNC-7 goal reported consuming three or more alcoholic drinks per day, a practice that can elevate BP. ${ }^{1,4}$ None of the participants above the JNC-7 goal pre-visit 1 reported use of NSAIDS on visit 4. All reported using acetaminophen for pain. Two of the three participants not at goal pre-visit 1 and who reported use of a decongestant, discontinued use by visit 4 .

Neither user age, nor years of education, nor REALM score were significantly correlated with BP, knowledge, self-efficacy, or adverse self-medication behavior risk score. The adverse self-medication behavior risk score was not significantly correlated with BP, knowledge, or self-efficacy scores.

\section{Satisfaction}

The overall mean satisfaction score was $4.21 \pm 0.30$ on the five-point, nine-item scale. Participants also indicated their degree of agreement with statements concerning their intent to change behaviors after using the PEP-NG. The overall mean intent to change score was $4.30 \pm 0.52$ on the five-point, six-item scale. All of the participants either agreed or strongly agreed with the statements "This program helped me want to change how I use medicines" and "This program helped me think of questions to ask my doctor." All of the participants either agreed or strongly agreed with the statement "After using this program I will make some changes in how I use medicines" and 10 (91\%) of the participants agreed with the statement "After using this program I will change when I take some medicines."

\section{Content analysis of qualitative interviews}

The first category identified from the interview transcripts was comfort with computer usage. All of the interviewed participants expressed being comfortable using computers prior to participating in the study. No changes in their attitudes or thinking toward computer usage occurred after using the PEP-NG. The participants described the PEP-NG as "easy to use", "self-explanatory", and "user friendly."

A second category, new medication knowledge, was evident among the participants. Four of the six (66\%) interviewed participants said they thought they understood their medication well before beginning the program. One participant acknowledged, "I don't understand them [medication purpose] all that well," while another claimed to understand their medications "perfectly" before beginning 
the program. The mixed sentiments of the participants indicate a disparity of knowledge prior to the study. All six participants stated the PEP-NG helped them learn about their medications. The participants enjoyed learning about their medication interaction and expressed interest in learning about the interactions of their other classes of medications. Most mentioned increase knowledge of medication interactions, with special concentration on NSAID pain relievers. One affirmed, "I learned that the Advil (ibuprofen) I was taking was interacting with my BP medication and now I take Tylenol (acetaminophen)." An improvement in knowledge related to alcohol intake and the negative effect on BP medication also was mentioned. One more medication the participants gained knowledge about was the ineffectiveness of Vitamin $\mathrm{E}$ in cardioprotection. $^{47}$

A third category was wariness of medication interactions. All participants said their knowledge of medication changed as a result of the PEP-NG program. Five out of six (84\%) said their views changed by learning about the interactions between their medications and supplements. The participants acknowledged they became "more wary" and aware of the conflicts between prescription drugs and supplements, as well as OTC drugs. One person noted:

"I think I'm more wary of how other things will interact with them. I mean just reaching up in the cabinet for OTC pain relievers. Inever thought OTC and prescription stuff were related in any way or form, or would be affected by the other so much. And now I don't take anything without saying, 'Wait a minute. Is there a problem?' There were a couple of surprises that I learned in that study that I shouldn't be taking, what would counteract what I would be taking for blood pressure medicine."

Another person said, "I changed some of my antacids, headache medication, or pain medication. I try to take Tylenol now instead of the other stuff."

The fourth category was making medication changes. Participants were asked if they changed the way they took their medicines after being in the study. Half said they already began or had started changing the time of day they were taking their medicine: "between the morning and night instead of all in the morning." One participant even noted a marked decrease in BP from changing the timing of the medication. Another mentioned waiting two hours after taking antihypertensives to take a supplement or an OTC medication in order to decrease the negative interactions. One participant changed taking antacids, "I would be popping Tums (calcium carbonate) at the wrong time of day...not even thinking about it. I was thinking 'Tums are Tums.' You can eat them like candy."

Changes or recommendations to improve the PEP-NG were queried. No major changes were recommended. Half stated they wanted more drug and dietary supplement choices to choose from on the user interface. Although the main focus was on drug/alcohol interactions with antihypertensive medications, several of the participants would have liked to learn how OTC drugs and alcohol influenced the medications they took for other health issues. Another change suggested by the participants involved the tutorial and animations. The PEP-NG software was originally designed for adults over the age of 60 and features a tutorial at the beginning of the user session. This sample, aged 45 to 60 years, found the tutorial repetitive. They enjoyed the animations, but recommended to make "the videos a little quicker for those of us who are more computer literate than others."

\section{Discussion}

Findings from this beta test of the PEP-NG in a worksite setting suggest high user satisfaction in the group aged 45-60 years. Participants also indicated that they were likely to make changes in their self-medication behaviors following use of the PEP-NG.

The PEP-NG had a medium effect size in increasing knowledge and a large effect in increasing self-efficacy for avoiding adverse self-medication behaviors. Knowledge scores were not significantly correlated with self-efficacy scores, a consistent finding in our previous studies with over 170 participants ${ }^{25-27}$ and expected if knowledge and self-efficacy are separate domains as theorized by Bandura. ${ }^{24}$ Behavior risk scores for medication errors decreased significantly. These results are similar to those from a previous beta test of the PEP-NG with 11 older adults aged 65-97 years. ${ }^{27}$ Historical control data with 60 older adult participants receiving face-to-face education only without the PEP intervention showed no changes in knowledge, self-efficacy, or behavior risk over time. ${ }^{25,26}$

All of the participants met the JNC-7 BP target by the end of the study suggesting that the intervention reported herein (that included monthly worksite BP checks, referral to the primary care provider when elevated BP readings were identified, and use of the PEP-NG program) can help identify workers not meeting BP goals and shed light on reasons for BP elevation such as poor adherence and/or adverse self-medication practices. This information can be shared (via the printout) with both the worker and the primary health care provider. 
A report for the Agency for Healthcare Research and Quality (AHRQ) documented mean reductions in systolic BP and diastolic BP as $4.5 \mathrm{~mm} \mathrm{Hg}$ and $2.1 \mathrm{~mm} \mathrm{Hg}$, respectively, across all studies and strategies examined ${ }^{48}$ The present study found $\mathrm{BP}$ reductions of over $5 \mathrm{~mm} \mathrm{Hg}$ for both systolic and diastolic BP for all six participants not meeting the JNC-7 goal upon entry to the study. Two of the six participants not at the JNC-7 goal on visit 1 had a provider-initiated change in their medication. Both of these participants had a BP of greater than $160 / 100$ on visit 1 and were referred immediately to their primary health care providers, who subsequently changed their antihypertensive regimens. Whether the elevated BP in these two participants would have been identified and attended to had the participants not been in the study is unknown. These results indicate that the PEP-NG intervention (which includes monthly BP measurements) at the worksite may offer a beneficial adjunct to the usual care of adults aged 45-60 years with poorly controlled hypertension.

The 1998-1999 Sloane telephone survey of a random sample of 2590 US adults reported $7 \%$ of adults aged 45-60 years were taking five or more prescription medications per day and $14 \%$ were taking five or more medications a day when OTC agents were included. ${ }^{49}$ Participants with hypertension in the current study took more daily medications than the general population of adults aged 45-60 years surveyed in the Sloane study. The differences may be due to sampling error given the small size of the present study. However, our prior studies also found greater self-medication use by older adults with hypertension compared to the general older population surveyed in the Sloane study. ${ }^{14,27}$

Measurement of patient medication adherence remains problematic. Blood assays and electronic adherence monitoring devices are costly and pharmacy refill rates and pill counts are labor-intensive. ${ }^{8,50,51}$ The cardiovascular risk associated with self-reported nonadherence (ie, answering a single survey question, "In the last month, how often did you take your medications as your doctor prescribed?") has been shown to be as great as that from smoking or diabetes. ${ }^{52}$ The simple self-report approach taken by the PEP-NG may underestimate adherence, but it does identify nonadherence when it is reported and may foster a subsequent discussion between patient and provider about the reasons for nonadherence as well as strategies for improved adherence to the medication regimen.

The PEP-NG was originally designed for the psychomotor skills and cognitive characteristics of older adults. The qualitative interviews reported herein are important because they support the usability of the PEP-NG in adults aged 45-60 years. These younger individuals with hypertension found the PEP-NG program engaging and informative. If themes such as "the program is boring" or "the program takes too much time" had been identified, the PEP-NG would need to undergo formal, iterative usability tests with this age group during extensive revision. Simple modifications were recommended: an expanded OTC and supplement database and a user-friendly speed adjustment for the animations.

The limitations of this pilot study (single worksite setting, student nurse providers, small, homogeneous Caucasian sample with high levels of health literacy, time series design without a control group or randomization, and self-reported medication use) prevent generalization to the population of adult workers with hypertension. However, the effect sizes attained are positive findings that support our plan to scale the PEP-NG intervention for a large, controlled effectiveness trial in a worksite setting.

\section{Conclusions}

The pilot data reported here suggest that the PEP-NG may help adult workers with hypertension and a high risk of PADI identify behaviors that they can change in order to improve adherence to their medication regimen and reduce adverse selfmedication behaviors. Our previous usability tests $(\mathrm{N}=48$ sets of observations) demonstrated that BP measurements did not change from the immediate pre- to the immediate postPEP use indicating that the PEP interface experience neither induced anxiety nor fostered relaxation. ${ }^{27,31}$ Thus, the PEP-NG appears to make good use of the user's waiting time. The improvements in knowledge, self-efficacy, self-medication behavior, and high satisfaction found in this pilot suggest that the PEP-NG could play a central role in worksite wellness programs aimed at workers with hypertension. Following revision of the animations to include a speed control in order improve the interface experience for younger adults, a large-scale controlled efficacy trial comparing the PEP-NG intervention with usual care in a nurse-run worksite wellness program is planned. If successful, both in terms of workflow and health outcomes, the PEP-NG could be an important asset in comprehensive employee health programs as described in the goals of the US Department of Health and Human Services document, Healthy People 2010. ${ }^{53}$

\section{Acknowledgments}

This study was funded through a Faculty Research Award to Patricia Neafsey from the University of Connecticut Research Foundation and through grants from the University 
of Connecticut Office of Undergraduate Research to Gregory Lutkus and Jessica Newcomb. The authors wish to thank Christian Rauh and Yan Li for creating the computer code for the PEP-NG interface, Zoe Strickler for animation design, Jonathan Gill for wireless network configuration and server support, and Sheri Peabody and Jessica Planas for their assistance in training the student nurse researchers. The authors are grateful to Kathlea Vaughn and Stephen Walsh for their advice on data analyses. The PEP-NG was developed with funding from the Ethel Donaghue Medical Research Foundation and the National Heart, Lung and Blood Institute, grant number 5R01HL084208 to Patricia Neafsey, Principal Investigator.

\section{References}

1. National Heart Lung, and Blood Institute. Healthy People 2010. Prevent and control America's high blood pressure: Mission possible. 2007. [cited 2007, Nov 7]. Available from: http://hp2010.nhlbihin. net/mission/abouthbp/abouthbp.htm.

2. Vasan RS, Beiser A, Seshardri S, et al. Residual lifetime risk for developing hypertension in middle aged women and men: The Framingham Heart Study. JAMA. 2002;287:1003-1010.

3. Berlowitz D, Ash A, Hickey E. Inadequate management of blood pressure in a hypertensive population. $N$ Engl J Med. 1998;339:1957-1963.

4. Chobanian AV, Bakris GL, Black HR, et al. Seventh report of the joint national committee on prevention, detection, evaluation and treatment of high blood pressure. Hypertension. 2003;42(6):1206-1256.

5. Düsing R. Adverse events, compliance, and changes in therapy. Curr Hypertens Rep. 2001;3:488-492.

6. Schroeder K, Fahey T, Ebraham S. How can we improve adherence to blood pressure-lowering medication in ambulatory care? A systematic review of randomized controlled trials. Arch Intern Med. 2004;164: 722-732.

7. Wong N, Lopez V, L'Italien G, Chen R, Kline SE, Franklin S. Inadequate control of hypertension in US adults with cardiovascular disease comorbidities in 2003-2004. Arch Intern Med. 2007;167(22): 2431-2436.

8. Osterberg L, Blaschke T. Adherence to medication. New Engl J Med. 2005;353(5):487-497.

9. Haynes RB, Ackloo E, Sahota N, McDonald HP, Yao X. Interventions for enhancing medication adherence. Cochrane Database Syst Rev. 2008;1:CD000011. DOI: 10.1002/14651858.CD000011.pub3.

10. Bosworth HB, Olsen MK, Goldstein MK, et al. The veterans' study to improve the control of hypertension (v-s.t.i.t.c.h.): Design and methodology. Contemp Clin Trials. 2005;26:155-168.

11. Roumie C, Elasy T, Greevy R, et al. Improving blood pressure control through provider education, provider alerts and patient education. A cluster randomized trial. Am J Intern Med. 2006;145:165-175.

12. Lee JK, Grace KA, Taylor AJ. Effect of a pharmacy care program on medication adherence and persistence, blood pressure, and low-density lipoprotein cholesterol: A randomized controlled trial. JAMA. 2006;296(21):2563-2571.

13. Institute of Medicine. Preventing Medication Errors. Washington, DC: National Academies Press; 2006.

14. Neafsey PJ, Shellman J. Adverse self-medication practices of older adults with hypertension attending blood pressure clinics. Internet $J A d v$ Nurs Pract. 2001;5(1):[about 10 p]. Available from: http://www.ispub. com/journal/the_internet_journal_of_advanced_nursing_practice/ volume_5_number_1_6/article/adverse_self_medication_practices_ of_older_adults_with_hypertension_attending_blood_pressure_ clinics_adverse_self_medication_practices.html.
15. Neafsey PJ, Jarrin O, Luciano S, Coffman M. Self-medication practices of Spanish-speaking older adults in Hartford, Connecticut. Hisp Health Care Int. 2007;5(4):169-179.

16. Gurwitz J, Field T, Harrold L, et al. Incidence and preventability of adverse drug events among older persons in the ambulatory setting. JAMA. 2003;289(9):1107-1116.

17. Wallsten S, Sullivan R, Hanlon J, Blazer D, Tyrey M, Westlund R. Medication taking behaviors in the high- and low-functioning elderly: MacArthur field studies of successful aging. Ann Pharmacother. 1995;29:359-364.

18. Aw TJ, Haas SJ, Liew D, Kurm M. Meta-analysis of cyclooxygenase-2 inhibitors and their effects on blood pressure. Arch Intern Med. 2005; 165:490-496.

19. Polonia J. Interaction of antihypertensive drugs with anti-inflammatory drugs. Cardiology. 1997;88(Suppl. 3):47-51.

20. MacDonald T, Wei L. Effect of ibuprofen on cardioprotective effect of aspirin. Lancet. 2003;361(9357):573-574.

21. Ho PM, Magid DJ, Shetterly SH, et al. Importance of therapy intensification and medication nonadherence for blood pressure control in patients with coronary disease. Arch Intern Med. 2008;168:271-276.

22. Peterson ED. Is information the answer for hypertension control? Arch Intern Med. 2008;168:259-260.

23. Bakris G, Ferdinand K, Douglas J, Sowers J. Optimal treatment of hypertension in African Americans. Postgrad Med. 2002;112(4): $73-82$.

24. Bandura A. Self-efficacy. Encyclopedia Human Behavior. 1994;4:71-81.

25. Neafsey PJ, Strickler Z, Shellman J, Padula A. Use of touchscreen equipped notebook computers to deliver health information about self-medication to older adults. J Gerontol Nurs. 2001;27(11):19-27.

26. Neafsey PJ, Strickler Z, Shellman J, Chartier V. An interactive technology approach to educate older adults about drug interactions arising from over-the-counter self-medication practices. Public Health Nurs. 2002;19(4):255-262.

27. Neafsey PJ, Anderson E, Peabody S, Lin C, Strickler Z, Vaughn K. Beta testing of a network-based health literacy program tailored for older adults with hypertension. Comput Inform Nurs. 2008;26(6):311-319.

28. Briesacher BA, Andrade SE, Fouayzi H, Chan A. Comparison of drug adherence rates among patients with seven different medical conditions. Pharmacotherapy. 2008;28(4):437-443.

29. Strickler Z, Neafsey PJ. Visual design of interactive software for older adults: Preventing drug interactions in older adults. Visible Language. 2002;36(1):4-28.

30. Strickler Z, Lin C, Rauh C, Neafsey PJ. Educating older adults to avoid harmful self-medication. Journal of Communication in Healthcare. 2008;1(1):110-128.

31. Lin CA, Neafsey PJ, Strickler Z. Usability testing by older adults of a computer-mediated health communication program. J Health Commun. 2009;14(2):102-118.

32. Lin CA, Neafsey PJ, Anderson E. APRN usability testing of a tailored computer- mediated health communication program. Comput Inform Nurs. 2010; In press.

33. Flesch R. A readability formula that saves time. J Reading. 1968; 11:129-228.

34. Department of Health and Human Services. Health insurance reform: Standards for electronic transactions: Announcemnet of designated standard maintenance organizations; final rule and notice No. 45 CFR Parts 160 and 162. Federal Register. 2003;68(34):8334-8374. Cited 2004, Sept 24. Available from: http://www.cms.hhs.gov/ SecurityStandard/Downloads/securityfinalrule.pdf

35. De Meyer F, Lundgren P, de Moor G, Fiers T. Determination of user requirements for the secure communication of electronic medical record information. Int J Med Inform. 1998;45:125-130.

36. International Organization for Standardization. ISO International Standards. 2004. [cited 2004 May 10]. Available from: http://www. iso.ch/iso/en/ISOOnline.frontpage.

37. Kelly M. What users want from a tool for analyzing and documenting electronic questionnaires. Soc Sci Comput Rev. 2000;18(4):407-420. 
38. Davis T, Long W, Jackson R, et al. Rapid estimate of adult literacy in medicine: A shortened screening instrument. Fam Med. 1993; 25:391-395.

39. Lawton MP, Brody EM. Assessment of older people: Self-maintaining and instrumental activities of daily living. Gerontologist. 1996;9:179-186.

40. Pfeiffer E. A short portable mental status questionnaire for the assessment of organic brain deficit in elderly patients. J Am Geriatric Soc. 1974;23(30):433-441.

41. Pickering TG, Hall JE, Appel LJ, et al. Recommendations for blood pressure measurement in humans and experimental animals: Part 1: Blood pressure measurement in humans: A statement for professionals from the subcommittee of professional and public education of the American Heart Association council on high blood pressure research. Hypertension. 2005;45:142-161.

42. Dunlop WP, Cortina JM, Vaslow JB, Burke MJ. Meta-analysis of experiments with matched groups or repeated measures designs. Psycho Methods. 1996;1:170-177.

43. Krippendorff K. Content Analysis: An Introduction to Its Methodology. 2nd ed. Thousand Oaks, CA: Sage Publications; 2004.

44. Isaksen SF, Jonassen J, Malone DC, Billups SJ, Carter BL, Sintek CD. Estimating risk factors for patients with potential drug-related problems using electronic pharmacy data. Ann Pharmacother. 1999;33(4): 406-412.

45. National Committee for Quality Assurance. HEDIS 2001. Washington, DC: NCQA: 2000.

46. Salerno SM, Jackson JL, Berbano EP. Effect of oral pseudoephedrine on blood pressure and heart rate. A meta-analysis. Arch Intern Med. 2005;165:1686-1694.
47. Collaborative Group of the Primary Prevention Project (PPP). Low-dose aspirin and vitamin $\mathrm{E}$ in people at cardiovascular risk: a randomized trial in general practice. Lancet. 2001;351:89-95.

48. Walsh J, McDonald KM, Shojania KG, et al. Hypertension care. In: Shojania KG, McDonald KM, Wachter RM, Owens DK, editors. Closing the Quality Gap: A Critical Analysis of Quality Improvement Strategies. Technical Review 9. (Prepared by the Stanford University-UCSF Evidence-based Practice Center, under Contract No. 290-02-0017). AHRQ Publication No. 04-0051-3. Rockville, MD: Agency for Healthcare Research and Quality; 2005.

49. Kaufman DW, Kelly JP, Rosenberg L, Anderson TE, Mitchell AA. Recent patterns of medication use in the ambulatory adult population of the united states. JAMA. 2007;287(3):337-344.

50. Choo PW, Rand CS, Inui TS, et al. Validation of patient reports, automated pharmacy records, and pill counts with electronic monitoring of adherence to antihypertensive therapy. Med Care. 1999;37(9): 846-857.

51. Krousel-Wood M, Thomas S, Munter P, Morisky D. Medication adherence: A key factor in achieving blood pressure control and good clinical outcomes in hypertensive patients. Curr Opin Cardiol. 2004;19(4):357-362.

52. Gehi AK, Ali S, Na B, Whooley MA. Self-reported medication adherence and cardiovascular events in patients with stable coronary heart disease. The Heart and Soul Study. Arch Intern Med. 2007;167:1798-1803.

53. United States Department of Health and Human Services. Healthy People 2010: Understanding and improving health and objectives for improving health. [cited 2009, May 5]. Available from: http://www. healthypeople.gov/.
Patient Preference and Adherence

\section{Publish your work in this journal}

Patient Preference and Adherence is an international, peer-reviewed, open access journal that focusing on the growing importance of patient preference and adherence throughout the therapeutic continuum. Patient satisfaction, acceptability, quality of life, compliance, persistence and their role in developing new therapeutic modalities and compounds to

\section{Dovepress}

optimize clinical outcomes for existing disease states are major areas of interest. This journal has been accepted for indexing on PubMed Central. The manuscript management system is completely online and includes a very quick and fair peer-review system. Visit http://www.dovepress.com/ testimonials.php to read real quotes from published authors. 